\title{
Review of: "Homeostasis"
}

Ulrich Pfeffer ${ }^{1}$

1 IRCCS Ospedale Policlinico San Martino

Potential competing interests: The author(s) declared that no potential competing interests exist.

Gordon Betts , cited on Wikipedia, gives the following definition of homeostasis: "In biology, homeostasis is the state of steady internal, physical, and chemical conditions maintained by living systems." (Gordon., Betts, J. Anatomy and physiology. DeSaix, Peter., Johnson, Eddie., Johnson, Jody E., Korol, Oksana., Kruse, Dean H., Poe, Brandon. Houston, Texas. p. 9. ISBN 9781947172043. OCLC 1001472383.). George E. Billmann delivers the following: "Homeostasis, as currently defined, is a self-regulating process by which biological systems maintain stability while adjusting to changing external conditions." (George E. Billmann, Homeostasis: The Underappreciated and Far Too Often Ignored Central Organizing Principle of Physiology. Front. Physiol., 10 March 2020, https://doi.org/10.3389/fphys.2020.00200).

Both definition appear more comprehensive to me than the one provided by David F. Marks. 